\title{
Unconditional Preparation of Squeezed Vacuum from Rabi Interactions
}

\author{
Hastrup, Jacob; Park, Kimin; Filip, Radim; Andersen, Ulrik Lund
}

Published in:

Physical Review Letters

Link to article, DOI:

10.1103/PhysRevLett.126.153602

Publication date:

2021

Document Version

Publisher's PDF, also known as Version of record

Link back to DTU Orbit

Citation (APA):

Hastrup, J., Park, K., Filip, R., \& Andersen, U. L. (2021). Unconditional Preparation of Squeezed Vacuum from Rabi Interactions. Physical Review Letters, 126(15), [153602]. https://doi.org/10.1103/PhysRevLett.126.153602

\section{General rights}

Copyright and moral rights for the publications made accessible in the public portal are retained by the authors and/or other copyright owners and it is a condition of accessing publications that users recognise and abide by the legal requirements associated with these rights.

- Users may download and print one copy of any publication from the public portal for the purpose of private study or research.

- You may not further distribute the material or use it for any profit-making activity or commercial gain

- You may freely distribute the URL identifying the publication in the public portal

If you believe that this document breaches copyright please contact us providing details, and we will remove access to the work immediately and investigate your claim 


\title{
Unconditional Preparation of Squeezed Vacuum from Rabi Interactions
}

\author{
Jacob Hastrup $\odot,{ }^{1, *}$ Kimin Park $\odot,{ }^{1,2, \dagger}$ Radim Filip $\odot,{ }^{2, \ddagger}$ and Ulrik Lund Andersen $\odot^{1, \S}$ \\ ${ }^{1}$ Center for Macroscopic Quantum States (bigQ), Department of Physics, Technical University of Denmark, \\ Building 307, Fysikvej, 2800 Kongens Lyngby, Denmark \\ ${ }^{2}$ Department of Optics, Palacky University, 77146 Olomouc, Czech Republic
}

(Received 2 July 2020; accepted 1 March 2021; published 13 April 2021)

\begin{abstract}
Squeezed states of harmonic oscillators are a central resource for continuous-variable quantum sensing, computation, and communication. Here, we propose a method for the generation of very good approximations to highly squeezed vacuum states with low excess antisqueezing using only a few oscillator-qubit coupling gates through a Rabi-type interaction Hamiltonian. This interaction can be implemented with several different methods, which has previously been demonstrated in superconducting circuit and trapped-ion platforms. The protocol is compatible with other protocols manipulating quantum harmonic oscillators, thus facilitating scalable continuous-variable fault-tolerant quantum computation.
\end{abstract}

DOI: 10.1103/PhysRevLett.126.153602

Introduction.-Quantum continuous variables have become an increasingly promising platform for quantum information processing [1]. In particular, extraordinary experimental progress has been made over the last few years in trapped-ion and superconducting circuit platforms toward fault-tolerant quantum computation [2-4]. One of the most promising routes toward fault-tolerant continuousvariable quantum computation is the Gottesman-KitaevPreskill encoding [5], which has gained substantial interest over the past few years due to experimental and theoretical developments $[4,6,7]$. For this encoding, highly squeezed states are an important resource for constructing highquality states $[6,8,9]$. The current record for squeezed vacuum is $15 \mathrm{~dB}$ [10] in an optical field using a parametric amplifier. However, non-Gaussian operations are difficult to realize efficiently in the optical regime, and thus it is challenging with current technology to further utilize this highly squeezed state for quantum computation.

On the other hand, qubit-coupled oscillators, such as a motional state of a trapped ion or a microwave cavity field coupled to a superconducting qubit can be manipulated with non-Gaussian operations via the qubit ancilla. In fact, universal control of the harmonic oscillator is in principle possible in such systems [11,12], although many protocols, such as squeezed state preparation, require specialized methods to be efficient. $12.6 \mathrm{~dB}$ squeezing has been reported in the motional state of a trapped ion [13] using a reservoir engineering technique [14]. This technique has the advantage of achieving squeezing in a steady-state configuration, thus facilitating the experimental implementation. However, the process utilizes spontaneous relaxation processes, the rates of which limit the speed at which the state is created and thus ultimately the achievable squeezing due to dephasing during the protocol. In the microwave regime $10 \mathrm{~dB}$ squeezing has been experimentally demonstrated [15]. This was achieved using a parametric amplifier realized by a metamaterial consisting of multiple Josephson junctions.

Here, we propose a method for the preparation of an approximate squeezed vacuum state in an oscillator strongly coupled to a qubit using only a few unitary interactions through the Rabi Hamiltonian [16,17]. This Hamiltonian has been experimentally demonstrated in trapped ions and superconducting circuits [4,18-20], and plays a key role in the Gottesman-Kitaev-Preskill encoding scheme of these platforms $[4,6,7]$. Thus the protocol facilitates the generation of highly squeezed states using a method that is compatible with further manipulation of the oscillator. Our protocol for the generation of squeezed vacuum is radically different from the common approach based on parametric amplification, and represents a fundamentally new view on squeezed vacuum generation. The obtainable amount of squeezing depends on the types and magnitude of noise in the particular system, but can generally be improved through faster interactions, e.g., through an increased power of the driving fields which control the interaction. Furthermore, the achievable amount of Fisher information is particularly robust against qubit errors during the protocol, making the generated states useful for sensing applications [21-27]. In particular, squeezed states can be used to detect displacements in the considered platforms using either the qubit coupling [28] or homodyne detection [29]. Finally, squeezed states serve as a fundamental resource for continuous-variable communication [1] which could find applications facilitating short-range connections in microwave circuits [30].

Protocol.-We consider a quantum harmonic oscillator described by the quadrature operators $\hat{X}$ and $\hat{P}$ satisfying $[\hat{X}, \hat{P}]=i$. In the vacuum state the oscillator exhibits equal fluctuations in each quadrature of magnitude 
$\left\langle\hat{X}^{2}\right\rangle=\left\langle\hat{P}^{2}\right\rangle=\frac{1}{2}$. The aim of the protocol is to generate a state squeezed in the $P$ quadrature by a relative amount $\Delta^{2}<1$ such that $\left\langle\hat{P}^{2}\right\rangle=\Delta^{2} / 2$. The protocol can straightforwardly be generalized to generate squeezing along an arbitrary quadrature direction, either by a rotation from the natural evolution of the squeezed state under a harmonic potential, or by a suitable change of quadrature operators during the protocol. It is common to quantify the squeezing in decibels relative to the vacuum as $\Delta_{\mathrm{dB}}=-10 \log _{10}\left(\Delta^{2}\right)$. A pure squeezed vacuum state, $\mid$ sqvac $\rangle_{\Delta}$, can be written in the $P-, X$ - and coherent state bases as

$$
\begin{aligned}
\mid \text { sqvac }\rangle_{\Delta} & \propto \int d p \exp \left(-\frac{p^{2}}{2 \Delta^{2}}\right)|p\rangle \\
& \propto \int d x \exp \left(-\frac{x^{2}}{2 \Delta^{-2}}\right)|x\rangle \\
& \propto \int d \alpha \exp \left(-\frac{\alpha^{2}}{\Delta^{-2}-1}\right)|\alpha\rangle,
\end{aligned}
$$

where the last line is only valid for $\Delta<1$ and the integral is over real $\alpha$. The coherent states with real $\alpha$ are defined as $|\alpha\rangle=e^{-i \sqrt{2} \alpha \hat{P}}|\operatorname{vac}\rangle \propto \int d x \exp \left[-(x-\sqrt{2} \alpha)^{2} / 2\right]|x\rangle$. For our approach, it is useful to view the squeezed state in the coherent state basis, as our strategy will be to directly construct a superposition of coherent states which resembles (1c). Since the coherent states form an overcomplete basis with large overlap between close-lying states, we can expect that Eq. (1c) holds to a good approximation even if we discretize the integral:

$$
|\mathrm{sqvac}\rangle_{\Delta} \underset{\sim}{\propto} \sum_{\alpha_{s} \in \mathfrak{R}} \exp \left(-\frac{\alpha_{s}^{2}}{\Delta^{-2}-1}\right)\left|\alpha_{s}\right\rangle,
$$

where $\mathfrak{Q}$ is a lattice on the real line. While the right hand side of Eq. (2) is technically a non-Gaussian state, we find that Eq. (2) is a good approximation for a lattice spacing of up to $\sim 1.5$, which is on the order of the width of a coherent state (details are presented in the Supplemental Material [31]). It is therefore possible to construct a highly squeezed state, practically indistinguishable from a Gaussian squeezed state, from a relatively sparse superposition of coherent states. A probabilistic method based on this approach was proposed in [32].

We now present a deterministic method to efficiently construct such a superposition of coherent states using a qubit ancilla. The circuit diagram of the protocol is shown in Fig. 1(a). We use two Rabi-type interaction Hamiltonians $\hat{P} \hat{\sigma}_{x}$ and $\hat{X} \hat{\sigma}_{y}[16,17]$, where $\hat{\sigma}_{x}$ and $\hat{\sigma}_{y}$ are the Pauli $x$ and $y$ operators of the qubit. Such Hamiltonians can be efficiently implemented [4,33-35], e.g., using a twotone drive which has been experimentally demonstrated in trapped ions $[18,19]$ or from a dispersive Jaynes-Cumming (a)

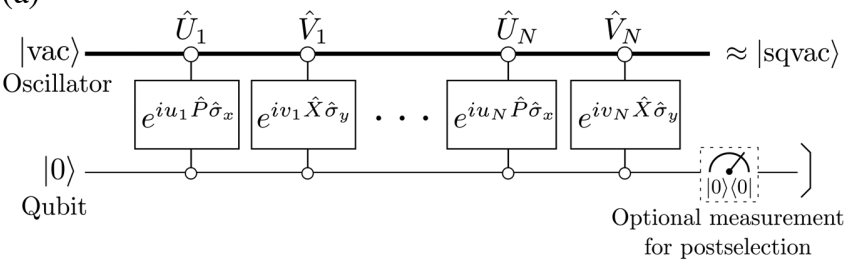

(b)
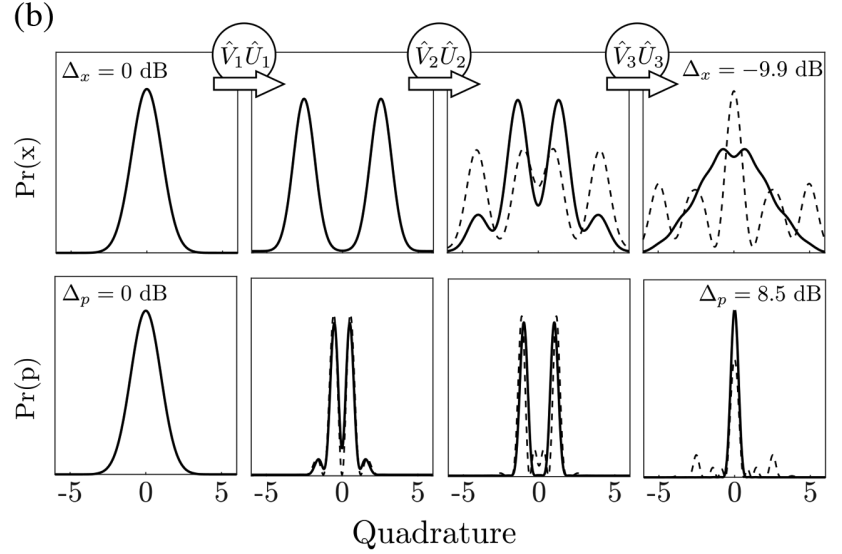

FIG. 1. (a) Circuit diagram for the generation of a $P$-squeezed vacuum state. The protocol consists of $N$ steps of interactions through the Hamiltonians $\hat{P} \hat{\sigma}_{x}$ and $\hat{X} \hat{\sigma}_{y}$. The interaction parameters $u_{k}$ and $v_{k}$ vary from step to step and are found numerically to optimize the protocol. The protocol is deterministic, but the performance can be slightly improved by measuring the qubit and postselecting on the outcome 0. (b) Evolution of the quadrature distributions during each step of the protocol. Dashed lines correspond to interaction parameters given by Eq. (3) with $L=0.45$ and solid lines correspond to numerically optimized interaction parameters. Note that the negative squeezing value in $\mathrm{dB}$ here denotes antisqueezing.

Hamiltonian which has been demonstrated in superconducting circuits $[4,20]$. The protocol consists of $N$ pairs of different interactions. The first type of interaction, $\hat{U}_{k}=\exp \left(i u_{k} \hat{P} \hat{\sigma}_{x}\right)$, displaces the oscillator in a direction depending on the state of the qubit, while the following interaction, $\hat{V}_{k}=\exp \left(i v_{k} \hat{X} \hat{\sigma}_{y}\right)$, approximately disentangles the qubit and the oscillator. The repeated application of these interactions creates a superposition of $2^{N}$ coherent states, and leaves the qubit back in the ground state. Note that the interactions used are conceptually similar to the protocol in Ref. [9] which aims to generate a grid state starting from squeezed vacuum. However, unlike this previous work, our target state consists of overlapping coherent states, for which the approximations used in Ref. [9] do not hold. In this work, we show that this limitation can be overcome surprisingly well by numerically optimizing the interaction parameters, $u_{k}$ and $v_{k}$, for each step, which enables the generation of squeezed states using Rabi interactions. Still, to understand why the protocol works and to have a good initial guess from which to optimize the interaction parameters, it is instructive to consider a specific set of interaction parameters given by 


$$
\begin{aligned}
& u_{k}= \begin{cases}2^{N-1} \sqrt{2} L, & \text { if } k=1, \\
-2^{N-k} \sqrt{2} L & \text { if } k>1,\end{cases} \\
& v_{k}= \begin{cases}2^{-(N-k)} \frac{\pi}{4 \sqrt{2} L}, & \text { if } k<N, \\
-\frac{\pi}{4 \sqrt{2} L} & \text { if } k=N,\end{cases}
\end{aligned}
$$

where $L$ is a free parameter, which determines the spacing of the resulting grid of coherent states. Each step aims to double the number of coherent states in the superposition. The first interaction $\hat{U}_{1}$ displaces the oscillator and entangles it with the qubit:

$$
\hat{U}_{1}|\mathrm{vac}\rangle|0\rangle=\frac{1}{\sqrt{2}}\left(\left|-2^{N-1} L\right\rangle|+\rangle+\left|2^{N-1} L\right\rangle|-\rangle\right) .
$$

Measuring the qubit in the $|0\rangle=(|+\rangle+|-\rangle) / \sqrt{2}$ or $|1\rangle=$ $(|+\rangle-|-\rangle) / \sqrt{2}$ state leaves the oscillator in a superposition of two coherent states, known as a Schrödinger's cat state, which has been experimentally demonstrated using exactly this type of interaction [20]. In our protocol, however, we do not require qubit measurements to disentangle the qubit and the oscillator. Instead, we apply a second interaction $\hat{V}_{1}$, which approximately disentangles the qubit and the oscillator,

$$
\begin{aligned}
\hat{V}_{1} \hat{U}_{1}|\mathrm{vac}\rangle|0\rangle= & \frac{1}{\sqrt{2}} \hat{V}_{1}\left(\left|-2^{N-1} L\right\rangle|+\rangle+\left|2^{N-1} L\right\rangle|-\rangle\right) \\
= & \frac{e^{-i \pi / 4}}{2}\left(\left[e^{-i \pi / 8}\left|-2^{N-1} L+i \frac{v_{1}}{\sqrt{2}}\right\rangle+i e^{i \pi / 8}\left|2^{N-1} L+i \frac{v_{1}}{\sqrt{2}}\right\rangle\right]|+i\rangle\right. \\
& \left.+\left[i e^{i \pi / 8}\left|-2^{N-1} L-i \frac{v_{1}}{\sqrt{2}}\right\rangle+e^{-i \pi / 8}\left|2^{N-1} L-i \frac{v_{1}}{\sqrt{2}}\right\rangle\right]|-i\rangle\right) \\
= & \frac{1}{\sqrt{2}}\left(\left|2^{N-1} L\right\rangle-\left|-2^{N-1} L\right\rangle\right)|1\rangle+\mathcal{O}\left(v_{1}\right),
\end{aligned}
$$

where $| \pm i\rangle=(|0\rangle \pm i|1\rangle)$ are the $\hat{\sigma}_{y}$ eigenstates, which are related to the $\hat{\sigma}_{x}$ eigenstates as $|+\rangle=e^{-i \pi / 4}(|i\rangle+i \mid-$ $i\rangle) / \sqrt{2}$ and $|-\rangle=e^{-i \pi / 4}(i|i\rangle+|-i\rangle) / \sqrt{2}$. In the last line we have used the relation $\langle\beta \mid \alpha\rangle=e^{i \operatorname{Im}\left(\beta^{*} \alpha\right)} e^{-|\beta-\alpha|^{2} / 2}$ to write $\left|-2^{N-1} L \pm i v_{1} / \sqrt{2}\right\rangle=e^{ \pm i \pi / 8}\left|-2^{N-1} L\right\rangle+\mathcal{O}\left(v_{1}\right)$, where $\mathcal{O}\left(v_{1}\right)$ denotes terms on the order $v_{1}$, which can be neglected when the coherent states are well separated, i.e., when $2^{N} L \gg 1$. Note that due to the complete absence of a measurement, the method does not rely on either postselection or active feed forward. Moreover, we circumvent accumulated measurement-induced noise such as measurement errors and bosonic noise. Each subsequent pair of interactions splits each coherent state into two, doubling the total number of peaks. Thus after all $N$ steps we produce the state

$$
\prod_{k}^{N} \hat{V}_{k} \hat{U}_{k}|\mathrm{vac}\rangle|0\rangle \approx\left(\sum_{s=0}^{2^{N}-1}\left|\left(2 s+1-2^{N}\right) L\right\rangle\right)|0\rangle,
$$

i.e., the oscillator ends in a superposition of multiple coherent states, similar to the target state of Eq. (2). However, there are two main issues with Eq. (6): first, the result yields an equal superposition of the coherent states, whereas our target state is convolved with a Gaussian envelope. Second, the approximation of Eq. (6) is only valid when the coherent states are sufficiently well separated, but to obtain a good approximation to a squeezed state, the coherent states need to be overlapping. It turns out that one can overcome these issues surprisingly well by tuning the interaction strengths of the protocol. This is illustrated in Fig. 1(b), showing the quadrature distributions of the oscillator for each step of the protocol. The solid lines represent the distributions using numerically optimized parameters while the dashed lines show the result for the parameters given by Eq. (3). Using the parameters of Eq. (3), the final $P$-quadrature distribution has side lopes which effectively reduces $\left\langle\hat{P}^{2}\right\rangle$ to that of vacuum. For the numerically optimized parameters, however, these lopes vanish, thus yielding a highly squeezed state. There are multiple reasons why the protocol is improved by tuning the interaction parameters. First, by tuning the strengths of the second interaction $v_{k}$, the qubit and the oscillator do not completely disentangle, so the subsequent controlled displacement $\hat{U}_{k+1}$ does not split each peak equally, but with a preferred direction, resulting in an unequal final distribution. This enables us to obtain an approximately Gaussian envelope over the resulting superposition as in Eq. (2). Additionally, as the states start to overlap, the disentangling interactions $\hat{V}_{k}$ have to be adjusted to optimize the disentanglement between the oscillator and qubit in the final step. Furthermore, when the coherent states of unequal amplitude are overlapping their peaks are effectively slightly shifted, which can be corrected by tuning the displacement interactions $\hat{U}_{k}$.

In Fig. 1(b) we have chosen the interaction parameters to optimize only the squeezing in the $P$ quadrature, yielding $\Delta_{p}=8.5 \mathrm{~dB}$ for $N=3$. The antisqueezing in the $X$ 
quadrature is slightly in excess, $\Delta_{x}=-9.9 \mathrm{~dB}$, due to the underlying non-Gaussianity of the state, but still quite close to the transform limit, showing that the output is a good approximation to a pure squeezed vacuum state. Still, even lower antisqueezing is possible without significantly compromising the squeezing by choosing the interaction parameters differently, e.g., one can obtain $\Delta_{p}=8.0 \mathrm{~dB}$ with $\Delta_{x}=-8.3 \mathrm{~dB}$ (see Supplemental Material [31]).

The resulting squeezing for the numerically optimized parameters is shown by the circles in Fig. 2. Only a few steps are required to generate a highly squeezed state, which is expected as the number of coherent states in the superposition increases exponentially with $N$. It is possible to further improve this result by roughly $1 \mathrm{~dB}$ by postselecting states for which the qubit is measured in the $|0\rangle$ state after all interactions. The protocol should leave the qubit in state $|0\rangle$ according to Eq. (6), but since this is an approximate result, a projection onto $|0\rangle$ helps improving this approximation. A postselectable result therefore also occurs with high probability. Note that while the produced states are fundamentally non-Gaussian, due to the finite and discrete number of underlying coherent states, the output is practically indistinguishable from a Gaussian squeezed stated. We confirm this in the Supplemental Material [31], showing that the generated states have fidelities of $>0.99$ with respect to pure Gaussian squeezed vacuum states.

From Fig. 2 we see that increasing the number of interactions monotonically increases the resulting squeezing. Thus the protocol can fundamentally be scaled to achieve large amounts of squeezing. However, real physical systems are affected by noise, such as dephasing and loss, which will accumulate during the protocol. Assuming the time for each interaction is proportional to the absolute interaction parameter, the total protocol duration roughly doubles each time $N$ is augmented, as the interaction parameters approximately scale as $2^{N}$ according to Eqs. (3). The increased squeezing therefore eventually gets counteracted by the accumulated noise. To study the effects

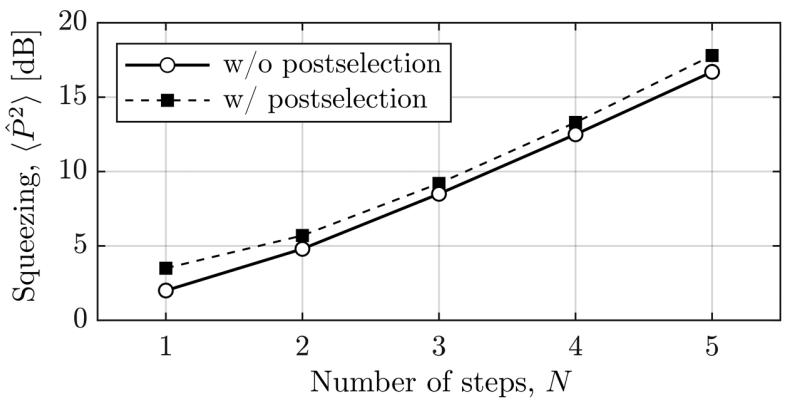

FIG. 2. Resulting squeezing as a function of the number of steps of the protocol. For each step, the squeezing increases with $\sim 3-4 \mathrm{~dB}$. The dashed curve corresponds to adding the optional measurement in Fig. 1(a) and postselecting on the outcome $|0\rangle$, which occurs with high probability. of noise, we simulate the protocol using the master equation,

$$
\frac{d \rho}{d t}=-\frac{i}{\hbar}[\hat{H}, \rho]+\hat{L} \rho \hat{L}^{\dagger}-\frac{1}{2}\left(\hat{L}^{\dagger} \hat{L} \rho+\rho \hat{L}^{\dagger} \hat{L}\right),
$$

where $\rho$ is the density matrix of the composite boson-qubit system. The Hamiltonian $\hat{H}$ is $\pm(\hbar / T) \hat{P} \hat{\sigma}_{x}$ or $\pm(\hbar / T) \hat{X} \hat{\sigma}_{y}$ during the two types of interactions, where the sign depends on the sign of the interaction parameter and $T$ is a timescale denoting the time required to implement $\exp \left(i \hat{P} \hat{\sigma}_{x}\right)$ or $\exp \left(i \hat{X} \hat{\sigma}_{y}\right)$. Thus the first interaction takes place in a time $T u_{1}$ after which the interaction Hamiltonian abruptly changes to the next one. $\hat{L}$ is the Lindblad noise operator, which determines the type of noise. We consider five types: (i) boson loss, $\hat{L}=\sqrt{\gamma} \hat{a}$; (ii) boson dephasing, $\hat{L}=\sqrt{\gamma}\left(\hat{a} \hat{a}^{\dagger}+\hat{a}^{\dagger} \hat{a}\right)$; (iii) boson heating [36], $\hat{L}_{1}=\sqrt{\gamma} \hat{a}, \hat{L}_{2}=\sqrt{\gamma} \hat{a}^{\dagger}$; (iv) qubit decay, $\hat{L}=\sqrt{\gamma}\left(\hat{\sigma}_{x}+i \hat{\sigma}_{y}\right) / 2$; and (v) qubit dephasing, $\hat{L}=\sqrt{\gamma} \hat{\sigma}_{z}$; where $\gamma$ is the noise rate and $\hat{a}=(\hat{X}+i \hat{P}) / \sqrt{2}$ is the bosonic annihilation operator. The results are shown in Fig. 3. For each noise source and noise rate we find that there exists an optimum number of interactions. Bosonic noise is seen to have a bigger impact compared to qubit noise. Especially, boson dephasing can heavily reduce the obtained squeezing, which is expected as squeezed states are generally sensitive to dephasing. The dashed lines show the outcomes which are postselected on measuring the qubit in state $|0\rangle$. We observe that the positive effect of postselection is now slightly larger compared to Fig. 2, especially for qubit-associated noise. This is because the noise can result in the qubit ending up in the $|1\rangle$ state, in which case the presence of noise can be detected and the event discarded. The postselection strategy can therefore effectively reduce the effect of noise.

A key property of squeezed states is their ability to detect displacements [21-23], which is quantified by the Fisher information [37], $I_{C}$. While the quadrature squeezing is affected by all noise types, the Fisher information turns out to be quite robust against qubit errors, as we show in the Supplemental Material [31]. For example, for $N=4$ with a qubit decay rate of $\gamma T=7 \times 10^{-1}$ we calculate $I_{C}=56$, which is equivalent to that of an $11.5 \mathrm{~dB}$ squeezed vacuum state. Thus the generated states can still be useful for sensing applications, even though they are generated under noisy conditions.

Finally, we benchmark our approach using noise figures from two recent experiments which implement exactly the types of interactions needed for our protocol in trapped ions [6] and microwave cavities [4]. For the parameters in [6] we calculate $9.3 \mathrm{~dB}$ squeezing and a Fisher information of $I_{C}=63$ (equivalent to a $11.9 \mathrm{~dB}$ squeezed state) using $N=4$. While this is slightly lower than the $12.6 \mathrm{~dB}$ reported in the trapped ion experiment in Ref. [13], we 


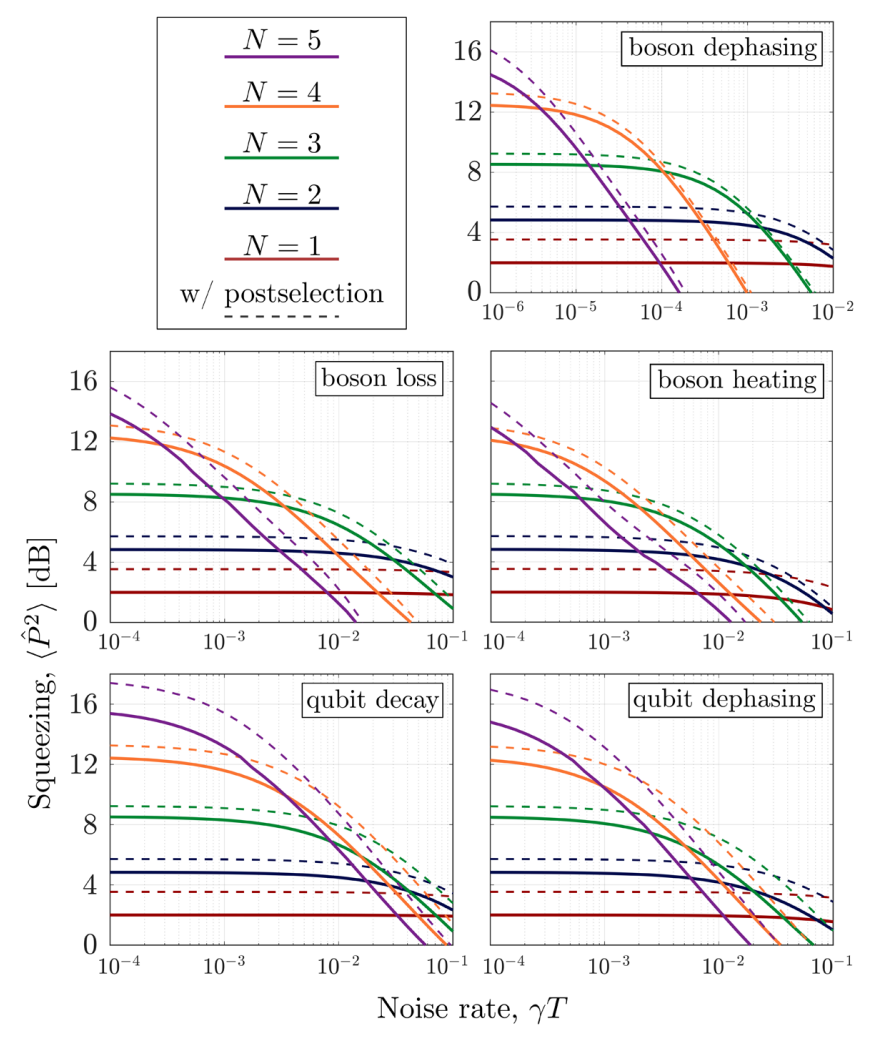

FIG. 3. Obtainable squeezing as a function of noise rate for different noise sources. For each noise source and noise rate, there exists an optimal number of steps. Postselecting on the outcome $|0\rangle$ can in some case improve the performance by more than $2 \mathrm{~dB}$.

point out that in their experiment the quadrature squeezing was not measured directly, but estimated using only the phonon population distribution. Since the quadrature squeezing is sensitive to small fluctuations and the coherence of the state, the $12.6 \mathrm{~dB}$ is likely overestimating the actual squeezing of the generated state. For the parameters in [4] we obtain an optimum squeezing of $7.0 \mathrm{~dB}$ at $N=3$, and an optimum Fisher information at of $I_{C}=86$ (equivalent to a $13.3 \mathrm{~dB}$ squeezed state) at $N=5$. The high Fisher information relative to the quadrature squeezing is due to the effect of qubit errors. In particular, these errors translate into non-Gaussian features of the output state which primarily degrade the quadrature squeezing (see Supplemental Material [31] for elaborate discussion).

Conclusion.-In conclusion, we have presented a deterministic protocol to produce a squeezed vacuum state via sequential application of two noncommuting Rabi Hamiltonians of the form $\hat{P} \hat{\sigma}_{x}$ and $\hat{X} \hat{\sigma}_{y}$. This interaction can currently be implemented via various methods in trapped-ion and circuit QED systems, but the protocol is not fundamentally limited to those systems and could be relevant for other qubit-oscillator platforms. Unlike previous methods, the protocol deterministically builds the squeezed state through a discrete superposition of coherent states. The protocol does not inherently require qubit measurements, but the performance can be slightly improved by postselecting on the state of the qubit. The possible amount of quadrature squeezing is ultimately limited by decoherence mechanisms of either the bosonic or qubit modes, while the achievable Fisher information is mainly limited by bosonic noise, and both can be improved by increasing the interaction strength and thus the speed of the protocol.

This project has received funding from the European Union's Horizon 2020 research and innovation programme under Grant Agreements No. 951737 and No. 731473. This project was supported by the Danish National Research Foundation through the Center of Excellence for Macroscopic Quantum States (bigQ, DNRF0142). R.F. acknowledges Projects No. LTAUSA19099 and No. 8C20002 from the Ministry of Education, Youth and Sports of Czech Republic. K. P. acknowledges Project No. 19-19722J of the Grant Agency of Czech Republic (GACR).

*jhast@fysik.dtu.dk

kimpa@fysik.dtu.dk

filip@optics.upol.cz

$\S$ ulrik.andersen@fysik.dtu.dk

[1] S. Braunstein and P. Van Loock, Quantum information with continuous variables, Rev. Mod. Phys. 77, 513 (2005).

[2] N. Ofek, A. Petrenko, R. Heeres, P. Reinhold, Z. Leghtas, B. Vlastakis, Y. Liu, L. Frunzio, S. Girvin, L. Jiang et al., Extending the lifetime of a quantum bit with error correction in superconducting circuits, Nature (London) 536, 441 (2016).

[3] L. Hu, Y. Ma, W. Cai, X. Mu, Y. Xu, W. Wang, Y. Wu, H. Wang, Y. Song, C.-L. Zou et al., Quantum error correction and universal gate set operation on a binomial bosonic logical qubit, Nat. Phys. 15, 503 (2019).

[4] P. Campagne-Ibarcq, A. Eickbusch, S. Touzard, E. Zalys-Geller, N. Frattini, V. Sivak, P. Reinhold, S. Puri, S. Shankar, R. Schoelkopf et al., Quantum error correction of a qubit encoded in grid states of an oscillator, Nature (London) 584, 368 (2020).

[5] D. Gottesman, A. Kitaev, and J. Preskill, Encoding a qubit in an oscillator, Phys. Rev. A 64, 012310 (2001).

[6] C. Flühmann, T. L. Nguyen, M. Marinelli, V. Negnevitsky, K. Mehta, and J. P. Home, Encoding a qubit in a trapped-ion mechanical oscillator, Nature (London) 566, 513 (2019).

[7] B. M. Terhal, J. Conrad, and C. Vuillot, Towards scalable bosonic quantum error correction, Quantum Sci. Technol. 5, 043001 (2020).

[8] B. M. Terhal and D. Weigand, Encoding a qubit into a cavity mode in circuit QED using phase estimation, Phys. Rev. A 93, 012315 (2016).

[9] J. Hastrup, K. Park, J. B. Brask, R. Filip, and U. L. Andersen, Measurement-free preparation of grid states, npj Quantum Inf. 7, 17 (2021).

[10] H. Vahlbruch, M. Mehmet, K. Danzmann, and R. Schnabel, Detection of $15 \mathrm{~dB}$ Squeezed States of Light and their Application for the Absolute Calibration of Photoelectric Quantum Efficiency, Phys. Rev. Lett. 117, 110801 (2016). 
[11] C. K. Law and J. H. Eberly, Arbitrary Control of a Quantum Electromagnetic Field, Phys. Rev. Lett. 76, 1055 (1996).

[12] S. Krastanov, V. V. Albert, C. Shen, C.-L. Zou, R. W. Heeres, B. Vlastakis, R. J. Schoelkopf, and L. Jiang, Universal control of an oscillator with dispersive coupling to a qubit, Phys. Rev. A 92, 040303(R) (2015).

[13] D. Kienzler, H.-Y. Lo, B. Keitch, L. De Clercq, F. Leupold, F. Lindenfelser, M. Marinelli, V. Negnevitsky, and J. Home, Quantum harmonic oscillator state synthesis by reservoir engineering, Science 347, 53 (2015).

[14] J. I. Cirac, A. A. Parkins, R. Blatt, and P. Zoller, "Dark" Squeezed States of the Motion of a Trapped Ion, Phys. Rev. Lett. 70, 556 (1993).

[15] M. Castellanos-Beltran, K. Irwin, G. Hilton, L. Vale, and K. Lehnert, Amplification and squeezing of quantum noise with a tunable Josephson metamaterial, Nat. Phys. 4, 929 (2008).

[16] P. Forn-Díaz, L. Lamata, E. Rico, J. Kono, and E. Solano, Ultrastrong coupling regimes of light-matter interaction, Rev. Mod. Phys. 91, 025005 (2019).

[17] A. F. Kockum, A. Miranowicz, S. De Liberato, S. Savasta, and F. Nori, Ultrastrong coupling between light and matter, Nat. Rev. Phys. 1, 19 (2019).

[18] C. Flühmann, V. Negnevitsky, M. Marinelli, and J. P. Home, Sequential Modular Position and Momentum Measurements of a Trapped Ion Mechanical Oscillator, Phys. Rev. X 8, 021001 (2018).

[19] D. Lv, S. An, Z. Liu, J.-N. Zhang, J. S. Pedernales, L. Lamata, E. Solano, and K. Kim, Quantum Simulation of the Quantum Rabi Model in a Trapped Ion, Phys. Rev. X 8, 021027 (2018).

[20] N. Langford, R. Sagastizabal, M. Kounalakis, C. Dickel, A. Bruno, F. Luthi, D. Thoen, A. Endo, and L. DiCarlo, Experimentally simulating the dynamics of quantum light and matter at deep-strong coupling, Nat. Commun. 8, 1715 (2017).

[21] V. Giovannetti, S. Lloyd, and L. Maccone, QuantumEnhanced Measurements: Beating the Standard Quantum Limit, Science 306, 1330 (2004).

[22] P. A. Ivanov and N. V. Vitanov, Quantum sensing of the phase-space-displacement parameters using a single trapped ion, Phys. Rev. A 97, 032308 (2018).

[23] M. Penasa, S. Gerlich, T. Rybarczyk, V. Métillon, M. Brune, J. M. Raimond, S. Haroche, L. Davidovich, and I. Dotsenko, Measurement of a microwave field amplitude beyond the standard quantum limit, Phys. Rev. A 94, 022313 (2016).

[24] P. A. Ivanov, N. V. Vitanov, and K. Singer, High-precision force sensing using a single trapped ion, Sci. Rep. 6, 28078 (2016).

[25] C. Hempel, B. Lanyon, P. Jurcevic, R. Gerritsma, R. Blatt, and C. Roos, Entanglement-enhanced detection of singlephoton scattering events, Nat. Photonics 7, 630 (2013).
[26] S. Burd, R. Srinivas, J. Bollinger, A. Wilson, D. Wineland, D. Leibfried, D. Slichter, and D. Allcock, Quantum amplification of mechanical oscillator motion, Science 364, 1163 (2019).

[27] J. B. Clark, F. Lecocq, R. W. Simmonds, J. Aumentado, and J. D. Teufel, Observation of strong radiation pressure forces from squeezed light on a mechanical oscillator, Nat. Phys. 12, 683 (2016).

[28] K. Duivenvoorden, B. M. Terhal, and D. Weigand, Singlemode displacement sensor, Phys. Rev. A 95, 012305 (2017).

[29] M. Naghiloo, D. Tan, P. M. Harrington, J. J. Alonso, E. Lutz, A. Romito, and K. W. Murch, Heat and Work Along Individual Trajectories of a Quantum Bit, Phys. Rev. Lett. 124, 110604 (2020).

[30] W. Pfaff, C. J. Axline, L. D. Burkhart, U. Vool, P. Reinhold, L. Frunzio, L. Jiang, M. H. Devoret, and R. J. Schoelkopf, Controlled release of multiphoton quantum states from a microwave cavity memory, Nat. Phys. 13, 882 (2017).

[31] See Supplemental Material at http://link.aps.org/ supplemental/10.1103/PhysRevLett.126.153602 for analysis of Eq. 2, fidelity of the generated states, discussion of excess anti-squeezing and analysis of the Fisher information generated states.

[32] K. Roszak, R. Filip, and T. Novotný , Decoherence control by quantum decoherence itself, Sci. Rep. 5, 9796 (2015).

[33] P. C. Haljan, K.-A. Brickman, L. Deslauriers, P. J. Lee, and C. Monroe, Spin-Dependent Forces on Trapped Ions for Phase-Stable Quantum Gates and Entangled States of Spin and Motion, Phys. Rev. Lett. 94, 153602 (2005).

[34] D. Ballester, G. Romero, J. J. García-Ripoll, F. Deppe, and E. Solano, Quantum Simulation of the Ultrastrong-Coupling Dynamics in Circuit Quantum Electrodynamics, Phys. Rev. X 2, 021007 (2012).

[35] A. Mezzacapo, U. Las Heras, J. Pedernales, L. DiCarlo, E. Solano, and L. Lamata, Digital Quantum Rabi and Dicke Models in Superconducting Circuits, Sci. Rep. 4, 7842 (2014).

[36] Boson heating is described by two Lindblad operators with strengths dependent on the temperature of the environment bath, $\hat{L}_{1}=\sqrt{\gamma_{c}(1+\bar{n})} \hat{a}$ and $\hat{L}_{2}=\sqrt{\gamma_{c} \bar{n}} \hat{a}^{\dagger}$, where $\gamma_{c}$ is the coupling rate to the bath with mean occupation number $\bar{n}$. Here, we define the heating rate $\gamma_{c} \bar{n} \equiv \gamma$ and assume $\bar{n} \gg 1$ such that $\gamma_{c}(1+\bar{n}) \approx \gamma_{c} \bar{n}$ to isolate the effect of heating rather than thermalization.

[37] M. Paris and J. Rehacek, Quantum State Estimation (Springer Science \& Business Media, New York, 2004), Vol. 649. 